\title{
MICROMORFOLOGÍA DE LA LEMA EN CATHESTECUM (POACEAE: CHLORIDOIDEAE: BOUTELOUINAE)
}

\author{
Darisol Pacheco Rivera, Patricia Dávila Aranda \\ Universidad Nacional Autónoma de México, Facultad de Estudios Superiores \\ Iztacala. Unidad de Biología, Tecnología y Prototipos (UBIPRO) \\ Av. de los Barrios s/n, Los Reyes Iztacala, 54090 Tlalnepantla, Edo. de México \\ darisolp@hotmail.com; pdavilaa@servidor.unam.mx \\ Jesús Valdés ReYna \\ Universidad Autónoma Agraria Antonio Narro, Departamento de Botánica \\ Buenavista, Saltillo, Coahuila \\ jvaldes@narro.uaaan.mx
}

\begin{abstract}
RESUMEN
Dada la utilidad taxonómica que presentan los caracteres de la epidermis de la lema y la escasez de información pertinente para el género Cathestecum, se examinan, describen y comparan estas características con aquellas presentadas por géneros afines de la subtribu Boutelouinae, con el fin de dilucidar sobre los posibles rasgos con valor taxonómico. Los resultados más significativos muestran la presencia de papilas en $C$. tamaulipense, $C$. erectum y C. brevifolium var. sonorense, la ausencia de aguijones y células de corcho en C. brevifolium y la ausencia de cuerpos de sílice en todas las especies de Cathestecum. Los géneros afines Buchloë, Soderstromia, Griffithsochloa, Pentarrhaphis y Bouteloua, representados en su mayoría por una sola especie, excepto Bouteloua y Pentarrhaphis, presentan abundantes papilas (no observadas en Bouteloua eludens ni en Griffithsochloa multifida) y células de corcho. Los cuerpos de sílice sólo se observaron en Buchloë dactyloides. Se concluye que algunas características micromorfológicas estudiadas pueden considerarse de valor taxonómico para separar las especies de Cathestecum, así como establecer, junto con otras fuentes de evidencia morfológicas, relaciones entre los taxa de la subtribu Boutelouinae.
\end{abstract}

Palabras claves: anatomía, Cathestecum, Gramineae, lema.

\begin{abstract}
Given the taxonomical utility of lemma epidermis characters and the scarcity of such information for the genus Cathestecum, these characteristics are examined, described and
\end{abstract}


compared with those of related genera in the subtribe Boutelouinae in order to elucidate possible characters of taxonomic value. The most significant results were the presence of papillae in C. tamaulipense, $C$. erectum and $C$. brevifolium var. sonorense, the absence of prickles and cork cells in C. brevifolium and the absence of silica bodies in all species of Cathestecum. Related genera (Buchloë, Soderstromia, Griffithsochloa, Pentarrhaphis and Bouteloua), each one represented by a single species (except Bouteloua and Pentarrhaphis), have abundant papillae (not observed in Bouteloua eludens and Griffithsochloa multifida) and cork cells. Silica bodies were observed only in Buchloë dactyloides. It is concluded that some of the micromorphological characters studied may be of taxonomic value for separating species of Cathestecum, as well as for establishing, along with others morphological sources, relationships among the taxa of subtribe Boutelouinae.

Key words: anatomy, Cathestecum, Gramineae, lemma.

\section{INTRODUCCIÓN}

El género Cathestecum J. Presl se incluye en la subfamilia Chloridoideae, la cual se distribuye substancialmente en las regiones cálido-secas del mundo. Fue descrito por primera vez en 1830 y su posición taxonómica a nivel de tribu y subtribu dentro de las gramíneas ha variado de acuerdo con diferentes apreciaciones. Clasificaciones más actuales ubican a Cathestecum en la tribu Chlorideae sensu lato (Watson y Dallwitz, 1992), mientras que Clayton y Renvoize (1986), Pohl en Davidse et al., (1994), así como Valdés y Dávila (1995) lo incluyen en la tribu Cynodonteae, subtribu Boutelouinae. Más recientemente, Columbus (1999) propone una nueva circunscripción taxonómica para definir la monofilia del grupo, en donde Cathestecum y otros otros géneros considerados como satélites, pasan a formar parte de Bouteloua.

En cuanto al número total de especies de Cathestecum, también ha habido cambios importantes basados en datos de diferentes fuentes, principalmente de tipo macromorfólogico (Vasey, 1884; Beal, 1896; Griffiths, 1912; Hitchcock, 1913, 1950; Swallen, 1937 y Pierce, 1978, 1979). En este estudio se sigue la propuesta de Pierce (1979), en la cual se reconocen e identifican cinco especies y cuatro variedades: $C$. brevifolium (var. brevifolium, var. hirsutum, var. ramosum y var. sonorense); C. erectum; C. prostratum; C. tamaulipense y $C$. varium. Actualmente, y con base en el trabajo de Columbus et al. (1998), se realiza una nueva revisión del género, incorporando otras fuentes de evidencia.

Cathestecum ha sido considerado como próximo a los géneros Buchloë, Buchlominus, Cyclostachya, Pentarrhaphis, Pringleochloa, Opizia y Griffithsochloa (Reeder y Reeder, 1963, 1966; Reeder et. al., 1965; Reeder, 1969; Rzedowski, 1975; Pierce, 1978). De acuerdo con Columbus et al. (1998), quienes estudiaron la filogenia de Bouteloua y géneros relacionados usando secuencias de ITS, Cathestecum se encuentra formando parte de un clado no resuelto junto a Bouteloua eludens, Buchloë, Griffithsochloa, Pentarrhaphis y Soderstromia. Estos últimos taxa han sido considerados en el presente estudio con el fin de establecer comparaciones con Cathestecum. 
En lo referente a la distribución geográfica de las especies de Cathestecum, éstas se encuentran desde el sur de Texas, en Estados Unidos, hasta México, Guatemala, Honduras y El Salvador, en Centroamérica. Su principal centro de diversidad es México, por lo que algunos autores consideran al género como endémico a este país y a Centroamérica (Swallen, 1937).

De manera general, a estas plantas se les observa creciendo en regiones de clima cálido, de relativamente baja precipitación y sometidas a menudo a condiciones de sequía severa. Algunas especies forman extensas áreas a la orilla de caminos y carreteras; en otros casos, constituyen el sustrato herbáceo dominante dentro de algunas comunidades vegetales y un recurso importante como forraje para consumo animal.

Como se mencionó anteriormente, los caracteres más utilizados en la delimitación de los taxa pertenecientes a este grupo han sido los macromorfológicos; sin embargo, en las gramíneas se ha demostrado la utilidad taxonómica de rasgos anatómicos a nivel de hoja (epidermis y corte transversal de lámina) y de la espiguilla. La epidermis de la lema, aunque menos estudiada que la de la lámina, ha facilitado la observación de dichos caracteres, tal como puede evidenciarse en diferentes estudios comparativos realizados (Terrell y Wergin, 1981, Terrell et al., 1983; Zuloaga y Sendulsky, 1988; Peterson, 1989; Valdés y Hatch, 1991; Columbus, 1996 y Snow, 1996). De estos trabajos, los cuatro últimos aportan información para taxa de Chloridoideae y en especial el de Columbus (1996), en el que se estudia la subtribu Boutelouinae. Por otro lado, Pierce (1979), en su trabajo biosistemático de Cathestecum y Griffithsochloa, describe los resultados de observaciones anatómicas de cortes transversales y epidermis de lámina, pero no incluye las relativas a las lemas.

Dada la poca información disponible sobre la micromorfología de la lema en el género Cathestecum, los objetivos de este estudio son: 1) examinar y describir las características anatómicas de la epidermis abaxial de la lema en las especies del mencionado género; 2) comparar estas características con aquellas presentadas por otros géneros afines de la subtribu Boutelouinae y 3) dilucidar sobre los posibles rasgos anatómicos con valor taxonómico.

\section{MATERIALES Y MÉTODOS}

Se removieron las lemas totalmente desarrolladas de ejemplares de diez especies pertenecientes a seis géneros de la subtribu Boutelouinae, incluyendo las cinco especies de Cathestecum (Apéndice 1). En su mayor parte, el material se obtuvo de colectas recientes y en los casos en que no se contaba con éste, se tomaron de ejemplares herborizados hace algún tiempo. Se seleccionó un promedio de tres especímenes, representativos del intervalo geográfico de cada especie. Las lemas fueron removidas del flósculo inferior de la espiguilla central de la rama, o de los flósculos fértiles femeninos para los taxa con espiguillas dimorfas (Buchloë dactyloides, Cathestecum brevifolium y Soderstromia mexicana). 
Posteriormente las lemas fueron fijadas en portamuestras de aluminio, con la ayuda de una cinta adhesiva de carbón conductivo y cubiertas durante 34 minutos con un baño de oro en un evaporador de vacío marca Desk II. Algunas de las lemas se trataron con xileno durante 30-45 minutos para remover la cera cuticular, la cual puede ocultar algunos elementos de la superficie. Sin embargo, al comparar los resultados con las lemas no tratadas, no hubo diferencias significativas.

Cada lema se examinó en un microscopio electrónico de barrido marca JEOL, modelo JSM-5310 LV, a un voltaje entre 10 y $20 \mathrm{kV}$ y a diferentes aumentos. En algunos casos, fue necesario el uso del microscopio esteroscópico para complementar y verificar la información obtenida. Las fotografías se tomaron utilizando placas fotográficas $4 \times 5$ pulg., Kodak TMax 100.

La superficie observada fue la epidermis abaxial, específicamente la porción media central situada entre las nervaduras. El lado adaxial de las lemas no presentó caracteres taxonómicos informativos, por lo cual no se incluyó en el estudio. Para la terminología empleada se siguió a Ellis (1976, 1979), Palmer y Tucker (1981) y la señalada en Anónimo (1962).

\section{RESULTADOS Y DISCUSIÓN}

En el Apéndice 2 se muestra el formato seguido para la observación y descripción de la epidermis abaxial de la lema, utilizando 28 caracteres en total. Con base en esta información y usando la terminología de los autores mencionados en el párrafo anterior, se presenta la descripción para las especies de Cathestecum.

Cathestecum brevifolium. Células largas intercostales: Paredes lisas a ligeramente onduladas en la var. brevifolium, ligeramente onduladas en las variedades hirsutum y sonorense. Papilas: Presentes en la var. sonorense, circulares o redondeadas, dorsales en las células largas, 1 por célula, en hileras horizontales (Fig. 1). Aguijones: Observados sólo en la var. sonorense, base elíptica, generalmente 1 aguijón por célula, en hileras horizontales (Fig. 1). Micropelos: Observados sólo en la var. brevifolium, bicelulares, claviformes, ápice redondeado, célula basal más larga que la distal, base contraída o estrecha (Fig. 2). Macropelos: No observados en la var. sonorense; unicelulares, escasos, con el ápice puntiagudo en las variedades ramosum y brevifolium (Fig. 2); con 2 o más células, abundantes, el ápice puntiagudo en la var. hirsutum (Fig. 3). Células de corcho: No observadas. Células de sílice: No observadas.

Cathestecum erectum. Células largas intercostales: Paredes ligeramente onduladas. Papilas: Circulares o redondeadas, dorsales en células largas, 1 por célula, en hileras horizontales (Fig. 4). Aguijones: Base elíptica, 1 aguijón por célula, en hileras horizontales (Fig. 4). Micropelos: Bicelulares, claviformes, el ápice 
ligeramente puntiagudo, la base estrecha (Fig. 5). Macropelos: Unicelulares, paredes delgadas, abundantes (Fig. 14). Células de corcho: Con forma de pesas vertical (más anchas o altas que largas) y bordes irregulares (Fig. 5). Células de sílice: No observadas.

Cathestecum prostratum. Células largas intercostales: Paredes lisas o ligeramente onduladas (Fig. 6). Papilas: No observadas. Aguijones: Base elíptica, 1 aguijón por célula, en hileras horizontales. Micropelos: Bicelulares, claviformes, ápice ligeramente puntiagudo, base estrecha. Macropelos: Unicelulares, paredes delgadas, frecuentes a abundantes. Células de corcho: Con forma de pesas vertical (más anchas o altas que largas) y bordes irregulares (Fig. 7). Células de sílice: No observadas.

Cathestecum tamaulipense: Células largas intercostales: Paredes medianamente onduladas. Papilas: Circulares o redondeadas, dorsales en las células largas, 1 por célula, arreglo irregular (presenta algunas zonas sin papilas) (Fig. 8). Aguijones: Base elíptica, 1 aguijón por célula, en hileras horizontales o de manera irregular. Micropelos: Bicelulares, claviformes, con el ápice redondeado y la base estrecha. Macropelos: Unicelulares, paredes delgadas, abundantes (Fig. 9). Células de corcho: Con forma de pesas vertical (más anchas o altas que largas) y bordes irregulares. Células de sílice: No observadas.

Cathestecum varium. Células largas intercostales: Cuadradas, con paredes moderadamente onduladas. Papilas: No observadas. Aguijones: No observados. Micropelos: Bicelulares, claviformes, con el ápice redondeado y base estrecha. Macropelos: No observados. Células de corcho: Con forma de pesas vertical (más anchas o altas que largas) y bordes irregulares (Fig. 10). Células de sílice: No observadas.

Seguidamente, se analizan de manera comparativa, las principales características de las lemas observadas en las especies de Cathestecum y en los taxa afines. Además, un resumen de estos resultados donde se confrontan taxa versus presencia/ausencia de estructuras anatómicas, se presenta en el Apéndice 3. Como detalle importante se puede mencionar que la abundancia de cera cuticular (incluso en las muestras tratadas con xileno), en muchos casos, dificultó la observación de algunas células. Es probable que el estudio de los patrones de distribución de esta cera pueda generar alguna información de utilidad taxonómica, tal como se ha reportado en algunos trabajos realizados en gramíneas (Palmer y Tucker, 1981; Terrel y Wergin, 1981).

Micropelos. Fueron observados en todos los taxa analizados, excepto en $C$. brevifolium variedades hirsutum, ramosum y sonorense. Se caracterizan por ser bicelulares, con la célula basal más larga y menos inflada que la distal. El ápice 
de esta última es redondeado, aunque existen formas ligeramente puntiagudas en $C$. erectum y $C$. prostratum.

Papilas. C. tamaulipense y C. brevifolium var. sonorense presentaron papilas circulares o redondeadas, con posición distal y arregladas en hileras horizontales. El número de papilas por célula larga fue de uno en la var. sonorense e irregular en $C$. tamaulipense, debido a que tenía zonas sin papilas. De los taxa afines a Cathestecum, se observaron estas estructuras en Buchloë dactyloides, Pentarrhaphis polymorpha y Soderstromia mexicana. En la primera especie las papilas son redondeadas, escasas y distribuidas de manera irregular (Fig. 11). En S. mexicana son abundantes, circulares o elípticas, arregladas en hileras (Fig.12). En $P$. polymorpha se destacan por su mayor tamaño, abundancia (más de una papila por célula larga), su posición central o distal y su arreglo irregular en las células largas (Fig. 13). Por último, en Griffithsochloa multifida y Bouteloua eludens no se observaron papilas.

Aguijones. No se encontraron aguijones en $C$. varium y $C$. brevifolium variedades brevifolium, hirsutum y ramosum. El resto de los taxa de Cathestecum presentan por lo general un aguijón por célula, con la base elíptica y distribuidos en hileras simples; sin embargo, $C$. tamaulipense exhibió algunas zonas sin aguijones. Asimismo, en B. eludens, B. dactyloides, G. multifida y P. polymorpha se presentaron estas estructuras distribuidas de manera irregular, siendo los aguijones más largos, abundantes y agudos en el último caso (Fig. 13). S. mexicana no presentó aguijones.

Macropelos. En C. varium y C. brevifolium variedades ramosum y sonorense, no se evidencia la presencia de macropelos, al igual que en $B$. dactyloides. Por el contrario, estas estructuras son abundantes en $C$. erectum, $C$. prostratum, $C$. tamaulipense, $C$. brevifolium var. hirsutum y $P$. polymorpha (Figs. 3, 9, 13 y 14); frecuentes (entre 3-10) en B. eludens y $G$. multifida y escasas (entre 1-2) en $C$. brevifolium var. brevifolium. Asimismo, con excepción de la var. hirsutum, donde se observaron macropelos unicelulares y multicelulares, en el resto de los taxa son exclusivamente unicelulares, con las paredes delgadas y el ápice puntiagudo (excepto en $P$. polymorpha donde son curvos, con paredes gruesas y el ápice es más agudo). Un detalle importante fue visto en $C$. tamaulipense, donde los macropelos son mucho más delgados con relación a los que se presentan en las otras especies (Fig. 9).

Células de corcho. Excepto en $C$. brevifolium, en todas las especies analizadas se observaron células de corcho. Éstas se presentan de manera solitaria y se caracterizan por ser más anchas o altas que largas, aplanadas, con paredes de bordes irregulares. La frecuencia de estas células es variable, pero por lo general son más abundantes hacia el ápice y márgenes de la lema. Columbus (1996) 
interpreta estas células como cavidades producto del colapsamiento de las paredes celulares. Del total de taxa analizados, $C$. varium fue la especie que presentó la mayor cantidad de células de corcho (Fig. 10).

Células de sílice. No se observaron células de sílice en ninguna especie de Cathestecum. De los taxa afines, sólo en $B$. dactyloides pudieron verse claramente estos cuerpos, con forma de silla de montar (saddle shaped) y acompañados de papilas (Fig. 11).

Células largas. En todos los casos analizados, excepto en $C$. varium que presentó una forma cuadrada, las células fueron rectangulares, es decir, más largas que anchas. La ondulación de las paredes fue variable, desde lisas en $C$. prostratum y en la var. brevifolium, ligeramente onduladas en $C$. erectum y $C$. tamaulipense, hasta moderadamente onduladas en $C$. varium y $C$. brevifolium var. sonorense. Paredes ligeramente onduladas se observaron en las especies afines, destacando las de B. eludens por ser ligeramente elevadas (Fig. 15).

\section{CONCLUSIONES}

Las características micromorfológicas examinadas y descritas del género Cathestecum, sugieren de manera general, que algunos de los rasgos anatómicos de la lema presentan poca variación entre las especies, especialmente los relacionados con las formas observadas en las diferentes estructuras. Sin embargo, resaltan características como las células largas intercostales cuadradas en C. varium, ausencia de papilas en $C$. prostratum y $C$. varium, papilas formando hileras horizontales en $C$. erectum y la distribución irregular de las papilas junto a macropelos más delgados en $C$. tamaulipense.

Con relación a $C$. brevifolium, un carácter distintivo observado fue la ausencia de células de corcho. De igual manera, se pueden apreciar rasgos que permiten distinguir las variedades, como son, la presencia de aguijones y papilas en la var. sonorense, la abundancia de macropelos bicelulares o multicelulares en la var. hirsutum, macropelos escasos en la var. brevifolium y zonas con ausencia de aguijones en la var. ramosum.

Por otro lado, características como las células de sílice (observadas sólo en Buchloë dactyloides), papilas de dos formas diferentes (Soderstromia mexicana) y de mayor tamaño (Pentarrhaphis polymorpha, B. dactyloides), macropelos curvos y de paredes gruesas ( $P$. polymorpha) y paredes de células largas ligeramente levantadas (Bouteloua eludens), son de significancia taxonómica para separar estos taxa dentro de la subtribu Boutelouinae. 


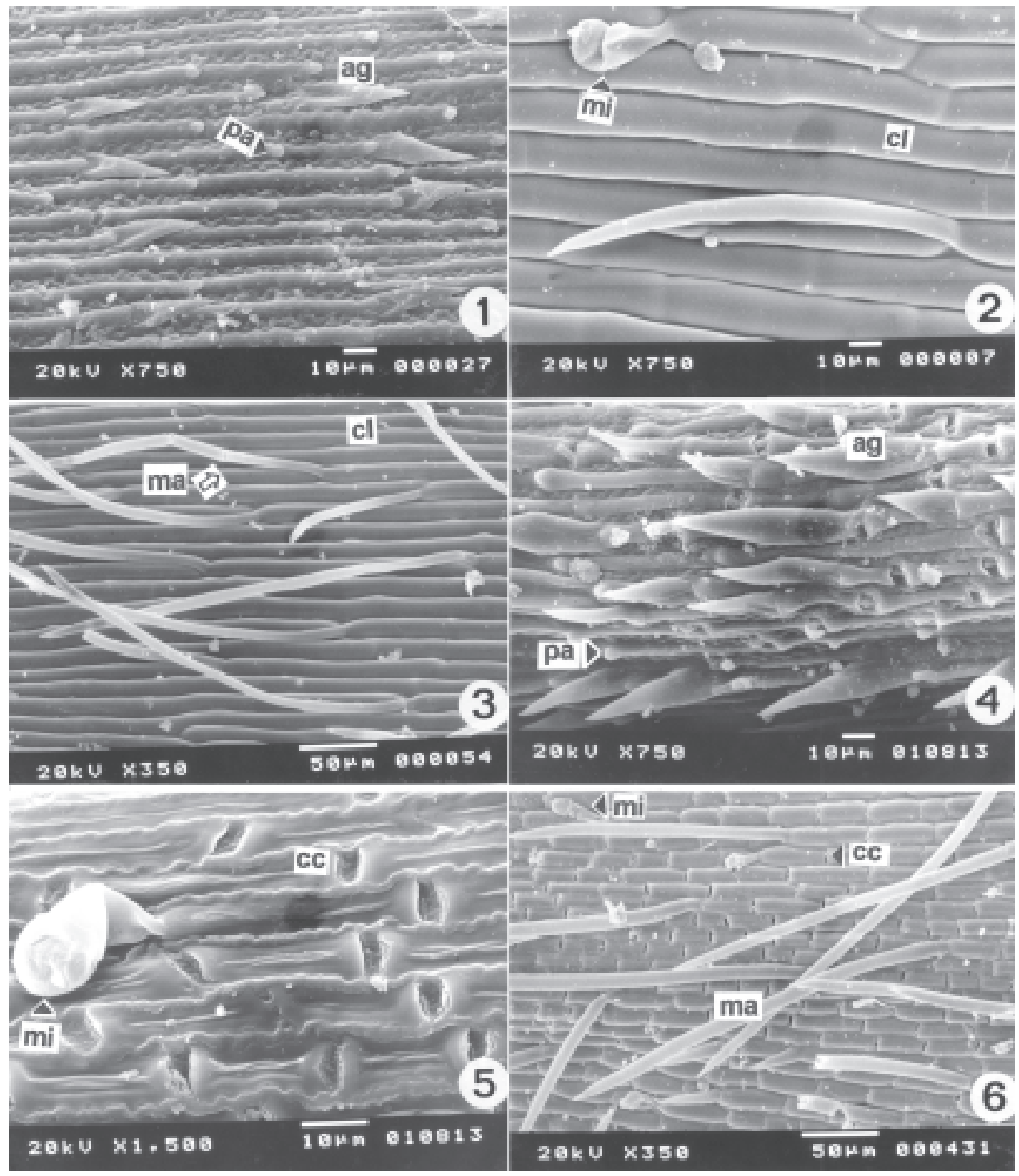

Figs. 1-6. Características micromorfológicas de la lema en Cathestecum: 1) C. brevifolium var. sonorense: papilas circulares y aguijones; 2) $C$. brevifolium var. brevifolium: paredes de células largas lisas y micropelo claviforme; 3) C. brevifolium var. hirsutum: abundantes macropelos; 4) C. erectum: papilas circulares y aguijones en hileras; 5$)$ C. erectum: células de corcho y micropelo claviforme; 6) C. prostratum: células de corcho, macropelos y aguijones. Abreviaturas: papilas (pa); aguijones (ag); macropelos (ma); micropelos (mi); células de corcho (cc); células largas (cl). 

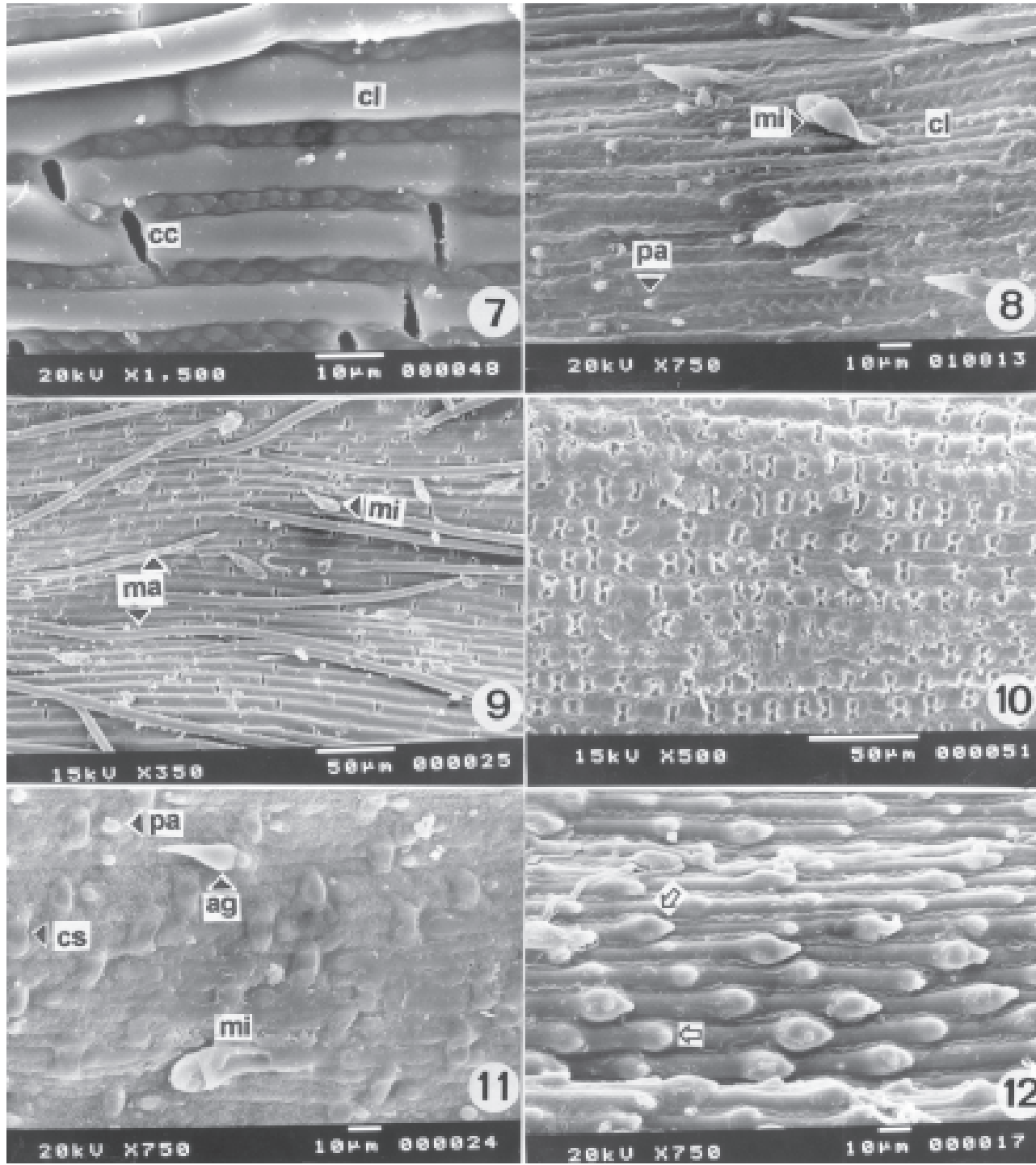

$20 k \cup \quad \times 758$
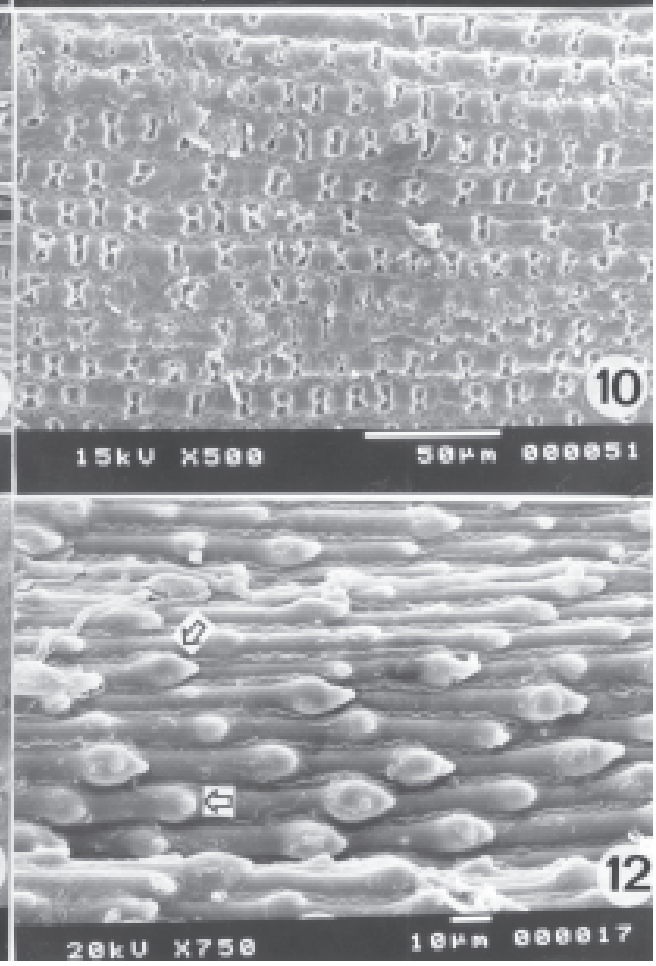

Figs. 7-12. Características micromorfológicas de la lema en Cathestecum y taxa afines: 7) C. prostratum: células de corcho altas y células largas; 8) C. tamaulipense: células largas con paredes medianamente onduladas, micropelos y papilas circulares; 9) $C$. tamaulipense: abundantes macropelos delgados y micropelos; 10) C. varium: abundantes células de corcho; 11) Buchloë dactyloides: cuerpos de sílice, papilas circulares, aguijones y micropelos; 12) Soderstromia mexicana: dos tipos de papilas. Abreviaturas: ver leyenda de las Figs. 1-6. 

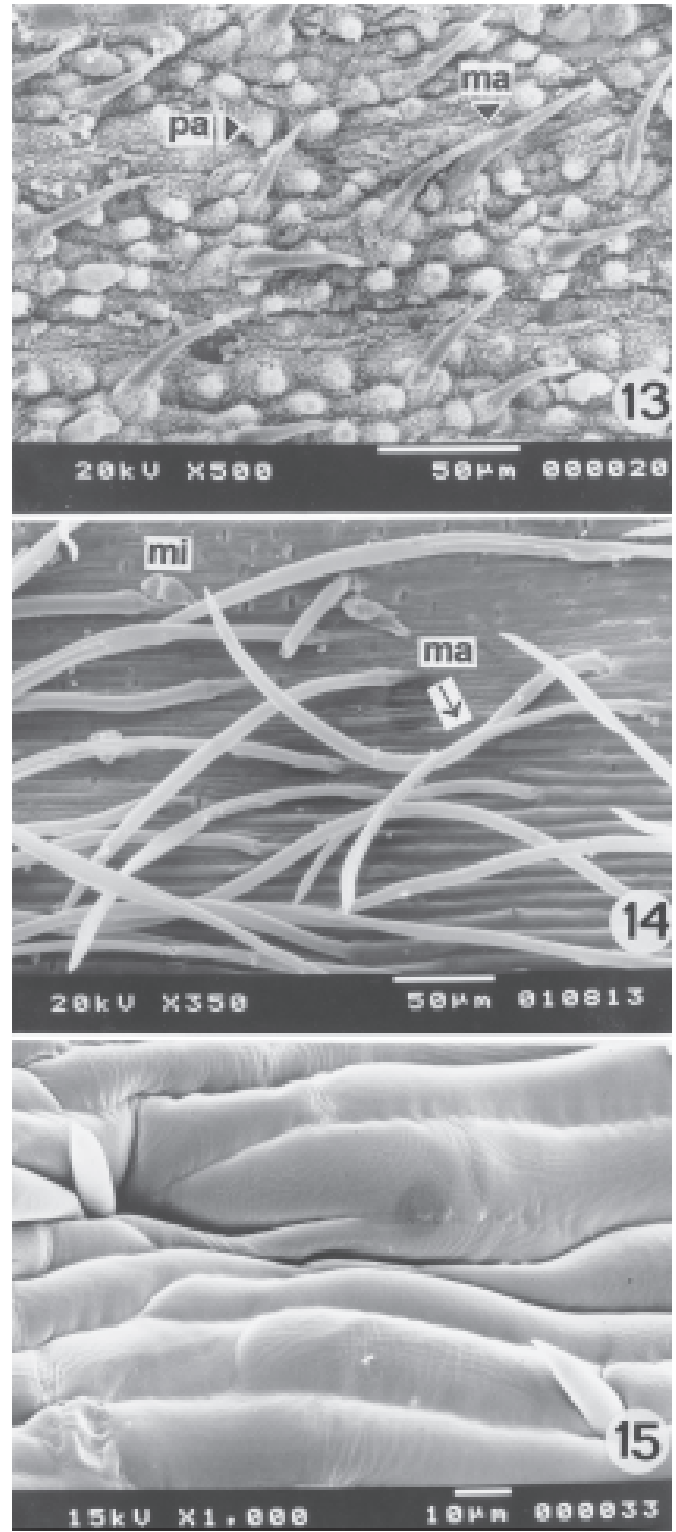

Figs. 13-15. Características micromorfológicas de la lema en Cathestecum y taxa afines: 13) Pentarraphis polymorpha: abundantes macropelos y papilas circulares; 14) C. erectum abundantes macropelos unicelulares; 15) Bouteloua eludens: células largas ligeramente levantadas. Abreviaturas: ver leyenda de las Figs. 1-6. 


\section{AGRADECIMIENTOS}

Agradecemos a la M. en C. Silvia Espinosa del Laboratorio de Microscopía Electrónica de la Facultad de Ciencias, Universidad Nacional Autónoma de México, por su asistencia técnica y sus valiosas sugerencias en la toma de las fotografías.

\section{LITERATURA CITADA}

Anónimo. 1962. II. Terminology of simple symmetrical plane shapes. Taxon 11: 145-156. Beal, W. J. 1896. Grasses of North America. Vol II. The grasses classified, described. Henry Holt \& Co. Nueva York. 706 pp.

Clayton, W. D. y S. A. Renvoize. 1986. Genera graminum: Grasses of the world. Kew Bull. Add. Ser. XIII. 389 pp.

Columbus, J. T. 1996. Lemma micromorphology, leaf blade anatomy and phylogenetics of Bouteloua, Hilaria and relatives (Gramineae: Chloridoideae: Boutelouinae). Ph. D. Dissertation. University of California. Berkeley. 258 pp.

Columbus, J. T. 1999. An expanded circumscription of Bouteloua (Gramineae: Chloridoideae). Aliso 18(1): 61-65.

Columbus, J. T., M. S. Kinney, R. Pant \& M. E. Siqueiros. 1998. Cladistics parsimony analysis of internal transcriber spacer region (nr DNA) sequences of Bouteloua and relatives (Gramineae). Aliso 17(2): 99-130.

Ellis, R. P. 1976. A procedure for standardizing comparative leaf anatomy in the Poaceae. I. The leaf blade as viewed in the transverse section. Bothalia 12(1): 65-109.

Ellis, R. P. 1979. A procedure for standardizing comparative leaf anatomy in the Poaceae. II. The epidermis as seen in surface view. Bothalia 12(4): 641-671.

Griffiths, D. 1912. The gramma grasses: Bouteloua and related genera. Contr. U. S. Natl. Herb. 14: 343-428.

Hitchcock, A. S. 1913. Mexican grasses in the United States National Herbarium. Contr. U. S. Natl. Herb. 17(3): 181-389.

Hitchcock, A. S. 1950. Manual of the grasses of the United States. Ed. 2. U. S. Dept. Agric. Misc. Pub. 200: 1-1051.

Holmgren, P. K., N. H. Holmgren y L. C. Barnett (eds.). 1990. Index Herbariorum, I. 8a. ed. New York Botanical Garden. Bronx, Nueva York. 692 pp.

Palmer, P. G. y E. Tucker. 1981. A scanning electron microscope survey of the epidermis of East African grasses. I. Smithsonian Contr. Bot. 49: 1-84.

Peterson, P. M. 1989. Lemma micromorphology in the annual Muhlenbergia (Poaceae). Southw. Naturalist 34(1): 61-71.

Pierce, G. J. 1978. Griffithsochloa, a new genus segregated from Cathestecum (Gramineae). Bull. Torrey Bot. Club 105:134-138.

Pierce, G. J. 1979. A biosystematic study of Cathestecum and Griffithsochloa (Gramineae). $\mathrm{Ph}$. D. Diss. University of Wyoming. Laramie. $244 \mathrm{pp}$.

Pohl, R. W. 1994. Cathestecum. In: Davidse, G., M. Sousa y A. Chater (eds.). Flora Mesoamericana. Vol. 6. Universidad Nacional Autónoma de México. México, D. F. pp. 296.

Reeder, J. R. 1969. Las gramíneas dioicas de México. Bol. Soc. Bot. México. 30: 121-126.

Reeder, J. R. y C. G. Reeder. 1963. Notes on Mexican grasses. II. Cyclostachya a new dioecious genus. Bull. Torrey Bot. Club 90: 193-201. 
Reeder, J. R. y C. G. Reeder. 1966. Notes on Mexican grasses. IV. Dioecy in Bouteloua chondrosoides. Brittonia 18: 188-191.

Reeder, J. R. y C. G. Reeder y J. Rzedowski. 1965. Notes on Mexican grasses. III. Buchlominus: another dioecious genus. Brittonia 17: 26-33.

Rzedowski, J. 1975. An ecological and phytogeographical analysis of the grasslands of Mexico. Taxon 24: 67-80.

Snow, N. 1996. The phylogenetic utility of lemmatal micromorphology in Leptochloa s. I. and related genera in subtribe Eleusininae (Poaceae, Chloridoideae, Eragrostideae). Ann. Missouri Bot. Gard. 83: 504-529.

Swallen, J. R. 1937. The grass genus Cathestecum. J. Wash. Acad. Sci. 27: 495-501.

Terrell, E. E. y W. P. Wergin. 1981. Epidermal features and silica deposition in lemmas and awns of Zizania (Gramineae). Amer. J. Bot. 68: 697-707.

Terrell, E. E., W. P. Wergin y S. A. Renvoize. 1983. Epidermal features of spikelets in Leersia (Poaceae). Bull. Torrey Bot. Club 110: 423-434.

Vasey, G. 1884. A new species of grass. Bull. Torrey Bot. Club 11: 37-38.

Valdés, J. y P. Dávila. 1995. Clasificación de los géneros de gramíneas (Poaceae) mexicanas. Acta Bot. Mex. 33: 37-50.

Valdés, J. y S. L. Hatch. 1991. Lemma micromorphology in the Eragrostideae (Poaceae). Sida 14(4): 531-549.

Watson, L. y M. J. Dallwitz. 1992. The grass genera of the world. Center for Agriculture and Biosciences. Wallingford. Reino Unido. 1038 pp.

Zuloaga, F. O. y T. Sendulsky. 1988. A revision of Panicum subgenus Phanophyrum section Stolonifera (Poaceae: Paniceae). Ann. Missouri Bot. Gard. 75: 420-455. 
Apéndice 1. Lista de especímenes utilizados en las observaciones micromorfológicas de la lema (abreviaturas según Holmgren et al., 1990)

Bouteloua eludens Griffiths

MÉxICo, Sonora: carretera Imuris - Cananea, km 109, al NW de Cananea, rancho El Jumate, $31^{\circ} 00^{\prime}$ lat. N, 110 $30^{\prime}$ long. W, $1200 \mathrm{~m}$ s.n.m., Pacheco y Zambrano 2560 (HERZU, IZTA, MEXU); carretera Cananea - Agua Prieta, aprox. $27 \mathrm{~km}$ al NE de Cananea, $31^{\circ} 05^{\prime}$ lat. N, $110^{\circ} 03^{\prime}$ long. W, $1400 \mathrm{~m}$ s.n.m., Pacheco y Zambrano 2561 (HERZU, IZTA, MEXU); ca. $41 \mathrm{~km} \mathrm{~S}$ of the International border at Nogales along Mexican Hwy 15, 1036 m s.n.m., Reeder y Felger 8127 (CHAPA).

Buchloë dactyloides (Nutt.) Engelm.

MÉxıco, Guanajuato: rancho La Mojada, $2 \mathrm{~km}$ de la comunidad de San José de Jofre, 2000 m s.n.m., González, s/n (XAL). México: mpio. Zempoala, Tepeyahualco, 2400 m s.n.m, Hernández 3267 (CHAPA). San Luis Potosí:

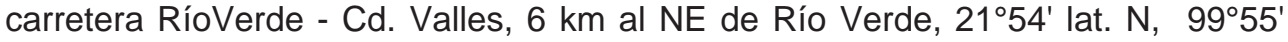
long. W, 950 m s.n.m., Pacheco y Zambrano 2551 (HERZU, IZTA, MEXU).

Cathestecum brevifolium Swallen var. brevifolium

MÉxIco, Guanajuato: carretera Irapuato - Silao, $19 \mathrm{~km}$ al $\mathrm{N}$ de Irapuato, $20^{\circ} 51^{\prime}$ lat. N, $101^{\circ} 18.336^{\prime}$ long. W, 1790 m s.n.m., Pacheco y Zambrano 2527, 2528 (HERZU, IZTA, MEXU). San Luis Potosí: carretera San Luis Potosí - Río Verde (Méx. 70), $45 \mathrm{~km}$ al W de Río Verde, $1 \mathrm{~km}$ a la entrada de San Juan Canoa, $22^{\circ} 01.090^{\prime}$ lat. $\mathrm{N}, 100^{\circ} 21^{\prime}$ long. W, 1160 m s.n.m., Pacheco y Zambrano 2550 (HERZU, IZTA, MEXU). Zacatecas: carretera Guadalajara - Zacatecas (Méx. 54),

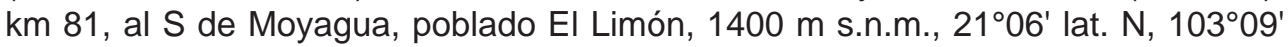
long. W, Pacheco y Zambrano 2573 (HERZU, IZTA, MEXU).

Comentario: Esta variedad es la que presenta el intervalo de distribución más amplio para el género. En México se registra para los estados de Aguascalientes, Colima, Puebla, Guanajuato, Jalisco, Morelos, Nayarit, San Luis Potosí, Sinaloa, Sonora y Zacatecas. Probablemente se extienda hacia el sur de Estados Unidos (Columbus, com. pers.), pero tal hecho no ha sido confirmado.

var. hirsutum G. J. Pierce, inéd.

El Salvador, mpio. Depto. de La Unión: El Carmen, $15-18 \mathrm{~km}$ al E de San Miguel, carretera San Miguel - La Unión, Linares y Herrera 4592 (HERZU). MÉxICO, Oaxaca: carretera Oaxaca - Tehuantepec (Méx. 190), 81 km de Oaxaca, vía Tehuantepec, $4.5 \mathrm{~km}$ de Totolapan, $16^{\circ} 42^{\prime}$ lat. N, 96¹9' long. W, 1000 m s.n.m., Pacheco y Zambrano 2591 (HERZU, IZTA, MEXU); carretera Tehuantepec - Oaxaca, km 204, 16²4' lat. N, 95³5' long. W, 100 m s.n.m., Pacheco y Zambrano 2592 (HERZU, IZTA, MEXU). 
Comentario: El centro de diversidad para este taxon se encuentra en el estado de Oaxaca, en particular entre la capital del estado y Tehuantepec. En Chiapas se registra de Tuxtla Gutiérrez, aunque no es abundante. La variedad marca el límite sur para el género, localizado en El Salvador, Centroamérica.

var. ramosum G. J. Pierce, inéd.

MÉxıco, Michoacán: carretera Apatzingán - Tepalcatepec, km 209, aprox. $6 \mathrm{~km}$ de Apatzingán, $1 \mathrm{~km}$ antes del Puente Las Majadas, $1^{\circ} 07^{\prime}$ lat. N, 102 ${ }^{\circ} 4^{\prime}$ long. W, 360 m s.n.m., Pacheco y Zambrano 2523 (HERZU, IZTA, MEXU); carretera (terracería) Apatzingán - Acahuato, aprox. $2 \mathrm{~km}$ de Apatzingán, al pie de Cerro El Húngaro, $19^{\circ} 07^{\prime}$ lat. N, 102 ${ }^{\circ} 1^{\prime}$ long. W, 540 m s.n.m., Pacheco y Zambrano 2525 (HERZU, IZTA, MEXU).

Comentario: Esta variedad sólo ha sido registrada de los alrededores de Apatzingán, en el estado de Michoacán, México y en algunos puntos del trayecto comprendido entre la mencionada localidad y Tepalcatepec, del mismo estado.

var. sonorense G. J. Pierce, inéd.

MÉxıco, Sonora: carretera Guaymas - Hermosillo, $7 \mathrm{~km}$ al N de Guaymas,

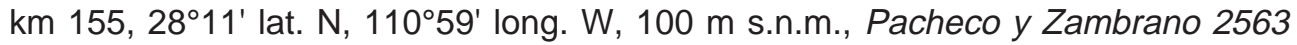
(HERZU, IZTA, MEXU); 400 km del desvío hacia Carbó, carretera hacia Santa Ana,

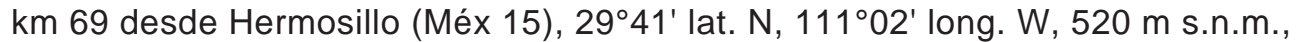
Pacheco y Zambrano 2554 (HERZU, IZTA, MEXU); carretera a la Bahía de San Carlos, $27^{\circ} 58^{\prime}$ lat. N, 11059' long. W, 20 m s.n.m., Pacheco y Zambrano 2564 (HERZU, IZTA, MEXU).

Comentario: La var. sonorense marca el límite norte para el género Cathestecum en México, en Magdalena de Kino, Sonora. El límite sur se encuentra en el estado de Sonora.

Cathestecum erectum Vasey \& Hack.

Estados Unidos: Texas: Presidio, $15-20$ mi S of Redford, Bofecillas Mts., Correll y Johnston 24437 (TEX); Chisos Mt area, Brewster Co, Warnock 1038 (TEX). MÉxIco: Coahuila: $33.5 \mathrm{~km}$ S of Boquillas on the Muzquiz Hwy not far from the Santa Rosa turnoff, $28^{\circ} 57^{\prime}$ lat. N, 10248' long. W, 960 m, Chiang y Wendt 9237 (MEXU).

Comentario: El principal centro de diversidad para esta especie es el suroeste de Texas, Estados Unidos, en la región conocida como Big Bend. Para México se registra también de Ojinaga, Chihuahua, en los límites con Presidio.

Cathestecum prostratum J. Presl

MÉxıco: Guerrero: carretera Puente Ixtla - Grutas, $4 \mathrm{~km}$ del entronque a Amacuzac ó $4 \mathrm{~km}$ al $\mathrm{N}$ de Michapa, 18³8' lat. N, 99²3' long. W, $1040 \mathrm{~m}$ s.n.m., Pacheco y Zambrano 2531 (HERZU, IZTA, MEXU); carretera Iguala - Chilpancingo, 
entrada a Zacacoyuya, $18^{\circ} 15^{\prime}$ lat. N, 99 ${ }^{\circ} 1^{\prime}$ long. W, 854 m s.n.m., Pacheco y Zambrano 2538 (HERZU, IZTA, MEXU); carretera hacia Xochipala, a $9.5 \mathrm{~km}$ del entronque carretera Chilpancingo - Iguala, $4 \mathrm{~km}$ de la estación de microondas de Xochipala ( $\mathrm{N}$ de Xochipala), $17^{\circ} 47^{\prime}$ lat. N, 99³6' long. W, $1100 \mathrm{~m}$ s.n.m., Pacheco y Zambrano 2540 (HERZU, IZTA, MEXU). Michoacán: carretera Cuatro Caminos - La Huacana, a $300 \mathrm{~m}$ del Puente El Naranjo, $13 \mathrm{~km}$ del entronque La Huacana - Nueva Italia, $18^{\circ} 54.731^{\prime}$ lat. N, $102^{\circ} 02^{\prime}$ long. W, 300 m s.n.m., Pacheco y Zambrano 2526 (HERZU, IZTA, MEXU). Oaxaca: $5 \mathrm{~km}$ al S de Huajuapan de León, vía Oaxaca, $17^{\circ} 46^{\prime}$ lat. N, 9745' long. W, 1520 m s.n.m., Pacheco y Zambrano 2584 (HERZU, IZTA, MEXU).

Comentario: Esta especie tiene su centro de diversidad en México, en los estados de Guerrero, Morelos, Michoacán, Oaxaca y Puebla. Es muy abundante en la cuenca del río Balsas.

Cathestecum tamaulipense G. J. Pierce, inéd.

Méxıco: Tamaulipas: carretera Santander Jiménez - San Fernando, $15 \mathrm{~km}$ al $\mathrm{N}$ de Santander Jiménez, aprox. $200 \mathrm{~m}$ de la entrada al Ejido El Encinal, 2423' lat. N, 98²3' long. W, 150 m s.n.m., Pacheco y Zambrano 2552 (HERZU, IZTA, MEXU); Santander Jiménez, Aldama cenotes, Valdés 2402 (ANSM).

Comentario: Fue propuesta por Pierce (1979) como una nueva especie dentro del género, sin embargo, su descripción no ha sido publicada. Se distribuye sólo en la localidad de Santander Jiménez, hacia el noreste de Ciudad Victoria, Tamaulipas, México.

\section{Cathestecum varium Swallen}

MÉxıco: Oaxaca: carretera Huajuapan de León - Tehuacán, km 71, 1 km de Huapanapan, $18^{\circ} 07^{\prime}$ lat. N, $97^{\circ} 40^{\prime}$ long. W, $1620 \mathrm{~m}$ s.n.m., Pacheco y Zambrano 2585, 2586 (HERZU, IZTA, MEXU); carretera Huajuapan de León - Tehuacán, km

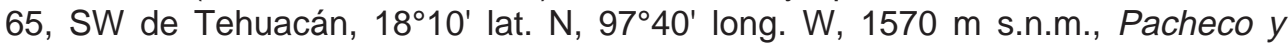
Zambrano 2588 (HERZU, IZTA, MEXU). Puebla: 5 km al NW de Ajalpan, 1300 m s.n.m., Medrano 1445 (COCA).

Comentario: Esta especie se conoce sólo del Valle de Tehuacán, y de áreas vecinas del estado de Oaxaca. Es más abundante hacia el suroeste de Tehuacán, tomando como referencia la carretera Huajuapan de León - Tehuacán.

Griffithsochloa multifida (Griffiths) G. J. Pierce

MÉxıco: Chihuahua: mpio. Bocoyna, San Juanito, Estrada $s / n$ (ANSM). Guerrero: carretera hacia Taxco, $20 \mathrm{~km}$ al NE de Taxco, entrada a Papala, $18^{\circ} 36^{\prime}$ lat. N, 99³2' long. W, 1520 m s.n.m., Pacheco y Zambrano 2533 (HERZU, IZTA, MEXU). Oaxaca: Autopista Nochixtlán - Oaxaca, aprox. $3 \mathrm{~km}$ del desvío a Nochixtlán, $17^{\circ} 24^{\prime}$ lat. N, $97^{\circ} 09^{\prime}$ long. W, 1840 m s.n.m., Pacheco y Zambrano 2590 (HERZU, IZTA, MEXU). 
Pentarrhaphis polymorpha (E. Fourn.) Griffiths

Méxıco, México: Cerro de la Cruz, 5 km al NW de Tepotzotlán, $2400 \mathrm{~m}$ s.n.m., Rzedowski 31963 (ENCB). Querétaro: carretera Amealco - San Juan del Río, aprox. $5 \mathrm{~km}$ al SE de Amealco rumbo a San Juan del Río, 20¹3' lat. N, 10005' long. W, 2320 m s.n.m., Pacheco y Zambrano 2547 (HERZU, IZTA, MEXU).

Soderstromia mexicana C. V. Morton

Méxıco, Guerrero: carretera Puerto Marqués - San Marcos (Méx. 200), a 13 km de San Marcos, dirección NW o 4 km del Puente La Estancia, 16 ${ }^{\circ} 46^{\prime}$ lat. N, 99²9' long. W, 90 m s.n.m., Pacheco y Zambrano 2541 (HERZU, IZTA, MEXU); carretera San Marcos - Cruz Grande, a 3 km al NW de Cruz Grande, entronque para Tierra Colorada, $16^{\circ} 43^{\prime}$ lat. N, 99 08' long. W, $70 \mathrm{~m}$ s.n.m., Pacheco y Zambrano 2542 (HERZU, IZTA, MEXU). 
Apéndice 2. Formato utilizado para examinar y describir la epidermis de la lema en Cathestecum y taxa afines.

Células largas intercostales

1) Forma de las células: rectangulares (1); cuadradas (2).

2) Ondulaciones en las paredes: paredes lisas, no onduladas (1); ligeramente onduladas (2); moderadamente onduladas (3); fuertemente onduladas (4).

Papilas

3) Papilas: ausentes o no observadas (1); presentes (2).

4) Forma de las papilas: ausentes (1); circulares o redondeadas (2); otra forma (3).

5) Distribución de las papilas en zona intercostal: ausentes (1); mayor de 50\%, con relación a células largas intercostales (2); menor de 50\% (3); dorsales en las células largas (4).

6) Número de papilas por célula larga intercostal: ninguna (1); una papila (2); más de una (3).

7) Posición de papilas: ninguna (1); central (2); distal (3).

8) Arreglo de papilas: ninguna (1); en una hilera horizontal (2); en dos o más hileras horizontales (3); irregular (4).

Aguijones

9) Aguijones: ausentes o no observados (1); presentes (2).

10) Base del aguijón: ninguna (1); elíptica (2); redondeada (3).

11) Número de aguijones por célula larga: ninguno (1); un aguijón por célula (2); más de un aguijón por célula (3); irregular, con zonas sin aguijones (4).

12) Arreglo de aguijones: ninguno (1); en una hilera simple (2); más de dos hileras (3); irregular (4).

Micropelos

13) Micropelos: ausentes o no observados (1); presentes (2).

14) No. de células en los micropelos: ninguna (1); bicelular (2).

15) Longitud de célula basal versus célula distal: ninguna (1); iguales (2); la basal más corta que la distal (3); la basal más larga que la distal (4).

16) Forma de célula basal versus célula distal: ninguna (1); ambas con la misma forma (2); la basal menos inflada que la distal (claviforme) (3); la basal más inflada que la distal (4).

17) Forma de célula distal: ninguno (1); ancho y largo iguales (2); largo menor que ancho (3); largo mayor que ancho (4).

18) Ápice de célula distal: ninguno (1); redondeado (2); puntiagudo (3).

19) Forma de la base: ninguna (1); estrecha (2); expandida (3).

20) Emergencia de la base: ninguna (1); formando ángulo con relación a la célula corta (2); en forma recta (3). 
Macropelos

21) Macropelos: ausentes o no observados (1); presentes (2).

22) No. de células de los macropelos: ninguna (1); una (2); dos (3); más de dos (4).

23) Paredes de los macropelos: gruesas (1); delgadas (2).

24) Abundancia de macropelos (visibles en campo de observación 100x o $1 \mathrm{~mm}$ ): ninguna (1); abundantes (más de 10 macropelos visibles) (2); frecuentes (entre 3-10) (3); escasos (entre 1-2) (4); irregulares (partes sin macropelos) (5).

Células de corcho y sílice

25) Células de corcho: ausentes (1); presentes (2).

26) Cuerpos de sílice: ausentes (1); presentes (2).

27) Forma de las células de corcho: ausentes (1); más altas que largas (1); más largas que altas (2); tan altas como largas (3).

28) Forma de las células de sílice: ausentes (1); con forma de silla de montar (2). 
Apéndice 3. Presencia (+) o ausencia (-) de características epidérmicas de la lema de Cathestecum y taxa afines. Abreviaturas: $C$. brevifolium (var. brevifolium $=\mathrm{CBr}$ br; var. hirsutum $=$ CBR-hi; var. ramosum $=$ CBR-ra; var. sonorense $=$ CBR-so); C. erectum $=\mathrm{CER} ; C$. prostratum $=\mathrm{CPR} ; C$. tamaulipense $=\mathrm{CTA} ; C$. varium $=$ CVA; Bouteloua eludens = BOU; Buchloë dactyloides = BUC; Griffithsochloa multifida = GRIF; Pentarrhaphis polymorpha $=$ PEN y Soderstromia mexicana $=$ SOD.

\begin{tabular}{|l|c|c|c|c|c|c|}
\hline Taxa & \multicolumn{7}{|c|}{ Carácter } \\
\hline & papilas & aguijones & macropelos & micropelos & $\begin{array}{c}\text { células } \\
\text { de corcho }\end{array}$ & $\begin{array}{c}\text { células } \\
\text { de sílice }\end{array}$ \\
\hline CBr-br & - & - & + & + & - & - \\
CBr-hi & - & - & + & - & - & - \\
CBr-ra & - & - & + & - & - & - \\
CBr-so & + & + & - & - & - & - \\
CER & + & + & + & + & + & - \\
CPR & - & + & + & + & + & - \\
CTA & + & + & + & + & + & - \\
CVA & - & - & - & + & + & - \\
BOU & - & + & + & + & + & - \\
BUC & + & + & - & + & + & + \\
GRIF & - & + & + & + & + & - \\
PEN & + & + & + & + & + & - \\
SOD & + & - & - & + & + & - \\
\hline
\end{tabular}

